\title{
Unstrittig und doch umstritten - europäische Solidarität in der Eurokrise
}

\author{
Heinz Kleger / Thomas Mehlhausen
}

\section{Indisputable and Still Disputed - European Solidarity During the Euro Crisis}

Abstract: The term solidarity is lacking a precise definition - despite being embedded as primary law in the EU treaties and regardless of its pivotal role in the current debate on the euro crisis. Once the semantic core is unearthed we sketch three dimensions of European solidarity (motives, references, level of action). Through this we shall distil three ideal types of an intergovernmental European solidarity: a federal, an organic and a distributive type. Empirically we assign the most relevant measures, which were discussed and partially enacted in the course of the euro crisis, to the three ideal types where we identify an increasing tendency towards a distributive solidarity. Once the first elements will be upgraded to a full-blown distributive solidarity through further integration, several risks arise from a normative perspective.

Keywords: Solidarity, Euro Crisis, European Union, Democracy, Justice

Schlagwörter: Solidarität, Eurokrise, Europäische Union, Demokratie, Gerechtigkeit

\section{Einleitung}

Im Zentrum des europaweiten Diskurses zur gegenwärtigen Eurokrise steht der Begriff der europäischen Solidarität. ${ }^{1}$ Gleich einem Wettstreit um die Interpretationshoheit über einen konstitutiven Gemeinschaftswert konkurrieren diverse Deutungen mit verschiedenen normativen Prämissen und distributiven Effekten, selbst wenn niemand den Wert an sich in Frage stellen würde. Dabei wird die finanzielle Notlage mehrerer Eurostaaten geradezu zum Lackmustest europäischer Solidarität stilisiert. Doch obwohl der Begriff der Solidarität in den primärrechtlichen Verträgen deutlich an Bedeutung gewonnen hat - gab es im Vertrag von Maastricht nur fünf Erwähnungen, rekurriert der Vertrag von Lissabon ganze $20 \mathrm{Mal}$ auf diesen Wert -, fehlt nach wie vor eine eindeutige Definition. Auch in der theoretischen Literatur mangelt es weitgehend an kohärenten Konzeptionen einer europäischen Solidarität zwischen den EU-Mitgliedstaaten (siehe aber Preuß 1998; Streeck 2000; Mau 2009). ${ }^{2}$

Eine solche Begriffsklärung ist jedoch aus mindestens drei Gründen vonnöten. Erstens ist für die politische Theorie eine exakte Definition eines derart zentralen Wertes deswegen essenziell, weil bei einer substanziellen Unterbestimmtheit diver-

1 Wir möchten insbesondere Andreas Busen, Thomas Fiegle und den beiden anonymen Gutachtern für ihre kritisch-konstruktiven Anmerkungen danken.

2 Aus Gründen der Lesbarkeit verwenden wir das Attribut „europäisch“ nicht im geografischen Sinne, sondern bezogen auf die Europäische Union. 
se Handlungen mit unterschiedlichen Verteilungsauswirkungen gerechtfertigt werden können. Dabei büßt dieser Wert wiederum an Legitimität ein, da die Bevorzugung einer Implementierungsvariante gegenüber einer anderen moralischpolitisch willkürlich wird (Chwaszcza 2008, S. 123). Zweitens können die im Beitrag vorgeschlagenen idealtypischen Solidaritätsmodelle der empirischen Politikwissenschaft zur Konzeptspezifikation (siehe Wonka 2007) und Theoriebildung (Papineau 1976) dienen, etwa für empirische Studien zur europäischen Öffentlichkeit (siehe Gerhards 1993; Eder et al. 1998; Risse 2002; Kantner 2004; Vetters, Jentges u. Trenz 2009). Drittens trägt eine klare Benennung von Bedingungen und Leistungen konkurrierender Solidaritätskonzepte im öffentlichen Diskurs dazu bei, disparate Handlungserwartungen offenzulegen, Missverständnissen vorzubeugen und mögliche Kompromisse zu identifizieren. Das Ziel des vorliegenden Beitrags ist somit, die diagnostizierte begriffliche Unschärfe zugunsten von drei Idealtypen aufzulösen, die sich in der gegenwärtigen Debatte zur Eurokrise wiedererkennen lassen.

Wir gehen in zwei Schritten vor. Im ersten Abschnitt klären wir den Begriff der europäischen Solidarität. Nachdem wir den semantischen Kern des Solidaritätsbegriffs herausgeschält haben, zeigen wir, dass das Verständnis einer europäischen Solidarität hinsichtlich der Motivation der solidarisch Handelnden, der Ausrichtung der Solidarleistungen und der Akteursebenen erheblich variieren kann. Da in der aktuellen Eurokrise auf diesen Gemeinschaftswert primär im Sinne einer zwischenstaatlichen Hilfsbereitschaft rekurriert wurde, konzentrieren wir uns auf den Typ der intergouvernementalen europäischen Solidarität und entwickeln dafür drei Idealtypen, die sich hinsichtlich der Motivation und Ausrichtung von Solidarakten unterscheiden. Im zweiten Teil des Beitrags ordnen wir die im Zuge der Eurokrise diskutierten und teils beschlossenen Maßnahmen den drei Idealtypen zu. Im Fazit werden abschließend die demokratietheoretischen Risiken einer sich immer stärker abzeichnenden distributiven europäischen Solidarität problematisiert.

\section{Europäische Solidarität: Entwirrung eines semantisch diffusen Begriffs}

\subsection{Zur Idee der Solidarität}

Der Begriff der Solidarität ist diffus, umstritten und ideengeschichtlich - in den Worten Herfried Münklers - „das Stiefkind der Moralphilosophie, aber auch der Gesellschaftstheorie“ (Münkler 2004, S. 15; vgl. auch Bayertz 1998a, S. 293; Fiegle 2002, S. 11). Nach unserer Auffassung lassen sich dennoch einige konstitutive Attribute aufzählen, die wir als semantischen Kern bezeichnen. ${ }^{3}$

Folgt man der Kurzdefinition von Steffen Mau als „Zusammenhang zwischen Individuen oder gesellschaftlichen Gruppen, der sich durch besondere Formen

3 Streng genommen sind alle politischen Begriffe per se umstritten und verweigern sich einer Reduzierung auf einen wie auch immer definierten semantischen Kern. Um jedoch nicht einem methodischen Relativismus zu verfallen, scheint es uns dennoch fruchtbar, zwischen den in der Literatur weniger umstrittenen Bedeutungsebenen und solchen zu differenzieren, in denen sich die Solidaritätsverständnisse erheblich unterscheiden (siehe dazu den nachfolgenden Abschnitt). 
von Verbundenheit und wechselseitiger Verpflichtung auszeichnet“ (Mau 2009, S. 63; siehe auch Honneth 1991), so muss erstens eine affektuelle Kohäsionskraft eines Wir-Gefühls bestehen, die zweitens eine Opferbereitschaft generiert, die deutlich über die Minimalleistungen eines nur humanitären Akts der Barmherzigkeit hinausgeht (Preuß 1998, S. 402). Solidarität ist zudem eine in der Regel freiwillige und stets latent reziproke zwischenmenschliche Handlungsdisposition. Freiwillig ist sie insofern, als dass der Rezipient Hilfeleistungen nicht rechtlich oder moralisch einfordern kann (Wildt 1998, S. 212; Thome 1998, S. 247). Reziprok ist sie nicht im juristischen Sinne analog zu einer Versicherung, sondern aufgrund der impliziten Erwartung, im Bedarfsfall selbst Solidarität zu erfahren (Thome 1998, S. 247; Schieder 2009, S. 18; Höffe 2002, S. 91).

Politische Solidarität - ursprünglich als Prinzip der Solidarhaftung ein Begriff aus der römischen Rechtslehre - ist ein fordernder und anspruchsvoller Wert. Umfang und Häufigkeit der Solidarleistungen können erheblich variieren, aber im Bedarfsfall kann ein solidarischer Beistand mit hohen Kosten für den Einzelnen verbunden sein. Darum wird eine solche auf einem belastbaren Gemeinschaftsgefühl basierende Hilfsbereitschaft durch die Bedingungen eingeschränkt, dass die eine Solidarleistung erzwingende Notlage als solche von Adressaten und Adressanten ähnlich wahrgenommen wird und der Rezipient bereits ernsthafte Anstrengungen zur Bewältigung dieser unternommen hat (Wildt 1998). Seine Intensität sinkt nicht nur (a) mit dem Grad der Selbstverschuldung der Notlage durch den Rezipienten und (b) mit wachsendem Umfang der notwendigen Solidarleistungen, sondern auch (c) mit der Größe der Gemeinschaft und einer damit einhergehenden abnehmenden Interaktionsdichte der beteiligten Individuen (Preuß 1998, S. 401; Voland 1998, S. 304; Wagener 2009, S. 78-79). In diesem soziologischen Sinne ist Solidarität aufgrund ihrer zeitlichen und räumlichen Kontingenz - im Gegensatz zur Barmherzigkeit oder Nächstenliebe - kein universalistischer Wert (vgl. Kersting 1998, S. 415; Bayertz 1998b, S. 21; Preuß 1998, S. 401; Schieder 2009, S. 20-21). ${ }^{4}$ Solidarität kann gleichwohl auch grenzüberschreitend gedacht werden, allerdings ist sie dann mit Grenzen der Motivation und Belastbarkeit konfrontiert. In welchem Sinne kann also vor diesem Hintergrund von einer spezifisch „europäischen“ Solidarität gesprochen werden?

\subsection{Dimensionen einer europäischen Solidarität}

Zunächst bezeichnet europäische Solidarität eine geografisch definierte Form der Solidarität, die sich im Diskurs zur Eurokrise konkret auf die Europäische Union (EU) bezieht. Jenseits des soeben beschriebenen semantischen Kerns kann eine europäische Solidarität hinsichtlich dreier Dimensionen variieren: der Motivation

4 Selbst die marxistische Formel der internationalen Solidarität war letztlich aus ideologiekritischer Sicht partikularistisch, da sie sich auf das unterdrückte Proletariat beschränkte (vgl. Göbel u. Pankoke 1998, S. 468). Zum (Spannungs-)Verhältnis zwischen Solidarität und Kosmopolitismus siehe Derpmann 2009, aber auch Bayertz 1998b, S. 11-12. Knüpft man Solidarität hingegen nur an eine allgemeine Anerkennung von Menschenrechten und nicht, wie wir, an eine (nicht existentialistisch verstandene) Opferbereitschaft, so wäre sicherlich auch eine synthetische anerkennungstheoretische Position (Taylor 1993) vertretbar. 
der solidarisch Handelnden, der Ausrichtung der Solidarleistungen sowie der Akteursebenen der Beteiligten.

Erstens können verschiedene Motivationen der solidarisch Handelnden identifiziert werden. Es lassen sich mindestens zwei moralische Rechtfertigungsgründe anführen, die ein gemeinwoblorientiertes solidarisches Handeln rechtfertigen. John Rawls argumentierte, dass aus der Interaktionsdichte der betroffenen Individuen eine gegenseitige moralische Verpflichtung aufgrund beidseitig vorteilhafter Kooperation entstünde (Rawls [1971] 1975, S. 20-23; siehe auch Durkheim [1893] 1977, S. 95-117). Daraus lässt sich ableiten, dass die Frage nach der Aufteilung der Kooperationsgewinne desto drängender wird, je intensiver die arbeitsteilige Kooperation von Menschen und somit auch je größer der Verflechtungsgrad der Mitglieder einer Solidargemeinschaft ist. Schließlich muss dann ein moralisches Kriterium diejenige der möglichen pareto-optimalen Aufteilungen bestimmen, die als fair gelten kann (Rawls [1971] 1975, S. 92-95). Nichtsdestotrotz kann selbst bei vollkommener Autonomie eine moralische Pflicht zu Ausgleichszahlungen mit dem Argument einer willkürlichen Verteilung von natürlichen Ressourcen innerhalb territorialer Grenzen verteidigt werden (Beitz 1979, S. 136ff.; Pogge 1994). Beide Argumente lassen sich angesichts des gemeinsamen Binnenmarktes und einer gewissen Ungleichheit von nationalen Rohstoffvorkommen auch auf die Europäische Union beziehen. ${ }^{5}$ Allerdings können Solidarakte in einem weiten Begriffsverständnis auch aus einer eigennutzorientierten Handlungsdisposition folgen (Baurmann 1998; Mau 2009). Das scheinbare Paradox zwischen Eigennutzorientierung und dem Solidaritätsbegriff innewohnender Gemeinwohlorientierung kann mit dem Hinweis darauf aufgelöst werden, dass bestimmte soziale Güter in arbeitsteilig organisierten Gesellschaften aufgrund einer funktionalen Interdependenz nur in Kooperation produziert werden können und mangels Handlungsalternativen so eine „Eigennutzsolidarität“ (Mau 2009, S. 67ff.) oder „instrumentelle Solidarität“ (Baum 1975) seitens der Gesellschaftsmitglieder erfordert.

Zweitens können ideengeschichtlich zwei einflussreiche Konzeptionen von Solidarität rekonstruiert werden, die sich in ihrer Ausrichtung erheblich unterscheiden. Horizontal ist die Solidarität, wenn sich die solidarisch Handelnden aufgrund ihrer ähnlich wahrgenommenen Lebensbedingungen zu einem gemeinsamen Zweck verbünden. In diesem Sinne fungierte der Solidaritätsbegriff der französischen Revolution - als „fraternité“ ursprünglich Brüderlichkeit meinend - und der deutschen Arbeiterbewegung als einigender Kampfbegriff, da sich Gleichgesinnte aufgrund ihrer vergleichbaren Lebensumstände zum Zweck einer Abwehr gemeinsamer Feinde miteinander zusammenschlossen (vgl. Bayertz 1998b; Wildt 1998). ${ }^{6}$ Vertikal ausgerichtet ist Solidarität dagegen dann, wenn die

5 Als wichtiger Indikator kann beispielsweise die Abhängigkeit der EU-Mitgliedstaaten vom Import von Energieträgern betrachtet werden. Während Malta zu 100 Prozent Energieträger importieren muss, weist Dänemark als einziger Mitgliedstaat einen Überschuss von 20 Prozent des eigenen jährlichen Verbrauchs auf, Eurostat 2011, S. 27.

6 Zum französisch-deutschen Begriffstransfer der Solidarität siehe Fiegle 2002. Zwar bezog sich der Begriff der „fraternité“ in der französischen Revolution auch auf unterdrückte „Brüder“, die (noch) nicht frei und gleich waren, doch ist er unserer Auffassung nach dennoch horizontal ausgerichtet, da die Adressanten solidarischer Hilfe die Adressaten als Gleiche wahrnahmen. 
wohlhabenderen den notleidenden Gemeinschaftsmitgliedern aufgrund eines Verbundenheitsgefühls beistehen (Bayertz 1998b). ${ }^{7}$ Dieser ideengeschichtliche Strang lässt sich auf die katholische Soziallehre zurückführen, denn hier gewährt der solidarisch handelnde Adressant dem Adressaten aufgrund seiner deutlich größeren Leistungsfähigkeit Beistand (Metz 1998, S. 190-191; Bayertz 1998b, S. 14). In Bezug auf die Europäische Union erkennt in diesem Sinne auch Ines Hartwig (2005, S. 166) eine „doppelte Dimension europäischer Solidarität“. Einerseits lösen die Mitgliedstaaten kollektiv als globaler Akteur externe Probleme, z. B. in Form einer gemeinsamen Handelspolitik oder im Rahmen der Gemeinsamen Sicherheits- und Verteidigungspolitik, und andererseits unterstützen die wirtschaftlich prosperierenden Mitglieder die schwächeren Staaten etwa in Form der Struktur- und Kohäsionsfonds.

Drittens kann das Attribut „europäisch“ den Begriff der Solidarität in Abhängigkeit von der jeweiligen Akteursebene in unterschiedlicher Weise geografisch konkretisieren. Eine intranationale Solidarität wäre dann europäisch, wenn eine Konvergenz der Wohlfahrtssysteme zu einer sukzessiven Homogenisierung von europaweiter Sozialstaatlichkeit führen würde, die sich aus globaler Perspektive als genuin europäisch charakterisieren ließe. ${ }^{8}$ Gøsta Esping-Andersen (1990) zeigt jedoch, dass sich drei unterschiedliche Wohlfahrtssysteme in Europa unterscheiden lassen: ein liberal-angelsächsisches, ein konservativ-kontinentaleuropäisches sowie ein sozialdemokratisch-skandinavisches. Im Zuge der Erweiterungen der EG bzw. EU verschärfte sich die Heterogenität mitgliedstaatlicher Sozialstaatlichkeit, sodass man durchaus neben einem südeuropäischen (Leibfried 1992; Ferrera 1996; Arts u. Gelissen 2002) auch ein postsozialistisches Wohlfahrtsmodell den drei westeuropäischen Typen hinzufügen könnte (Fenger 2007; Aidukaite 2009; kritisch aber Bazant u. Schubert 2008, S. 644). Eine europäische Solidarität im Sinne ähnlich praktizierter, staatlich koordinierter Sozialpolitiken existiert daher allenfalls rudimentär.

Eine transnationale Solidarität lässt sich hingegen immerhin ansatzweise in der EU erkennen (Hühn et al. 2010). Als grenzüberschreitende nichtkommerzielle Kooperation zivilgesellschaftlicher Akteure ist eine solche Solidarität zwar beispielsweise im gewerkschaftlichen Bereich in Form des Europäischen Gewerkschaftsbundes (Buschak 2003), der Europäischen Betriebsräte (Jagodziński 2007) oder des Europäischen Sozialforums im Entstehen, doch eine substanzielle Bedeutung kann diesen Institutionen nur bedingt zugesprochen werden (kritisch dazu:

7 In seiner ideengeschichtlichen Spurensuche nach den Bedeutungsebenen dieses Begriffs identifiziert Bayertz vier relevante Strömungen, wobei er für nur zwei, unserer referentiellen Differenzierung durchaus ähnelnde Konzeptionen plädiert: Kampf-Solidarität und Gemeinschafts-Solidarität.

8 Nun kann in Frage gestellt werden, ob Sozialstaatlichkeit tatsächlich Ausdruck von Solidarität ist. Einerseits mag kritisiert werden, dass bei einer solchen institutionell vermittelten „administrativen Solidarität“ die Zielgruppe nicht mehr ausschließlich die Bedürftigen sind (Schuyt 1998, S. 301). Andererseits kann argumentiert werden, dass eine staatlich erzwungene Hilfsleistung die solidarische Motivation erstickt (Halldenius 1998, S. 339). Wir plädieren dennoch dafür - ob nun nationalstaatlich oder supranational koordinierte - Sozialstaatlichkeit als institutionell vermittelte und verrechtlichte Solidarität zu fassen, da Solidarität nicht nur der Ursprung der Sozialstaatlichkeit war, sondern auch als gesellschaftlicher „Kitt“ eine funktionale Voraussetzung für ihre Akzeptanz und Fortbestand darstellt. 
Streeck 2000; Zürcher 2001; Deppe 2005; Bieler 2007; Traum 2005, S. 96). Aufgrund der Mobilisierungskosten werden transnationale Solidarakte, z. B. in Form von Protestaktionen oder Beistand bei Naturkatastrophen, eher punktuell und mit geringer Nachhaltigkeit geleistet. Es darf dennoch vermutet werden, dass diese Dimension der europäischen Solidarität bei fortschreitender Vertiefung als kohäsionsförderndes Element an Bedeutung gewinnen wird (Heidenreich 2007; Magnusson u. Stråth 2007), je mehr soziale Problemthemen in einem europäischen Kontext wahrgenommen werden.

Eine supranationale Solidarität steht im Zentrum der Debatte um die Wünschbarkeit eines sozialen Europas (siehe Scharpf 2002, 2009; Begg 2005; Stephan 2005; Ganßmann 2010; Lessenich 2010; Scharpff 2012). Institutionalisierte und auf supranationale Ebene delegierte Solidarmaßnahmen zielen auf eine Angleichung der innereuropäischen Lebensbedingungen. Da in der Nachkriegszeit der europäische Nationalstaat soziale Rechte vor dem ökonomischen Wettbewerbsdruck geschützt hat, müssen aufgrund der negativen europäischen Integration nun auch supranationale Korrekturmechanismen hinzutreten (Streeck 2000, S. 245). Aufgrund der distributiven Konsequenzen einer Supranationalisierung nationaler Sozialpolitiken gab es in diesem Bereich bisher nur sehr beschränkte Kompetenzübertragungen auf die europäische Ebene. Dennoch existieren in Form von allgemeinen Rechtsstandards und verschiedenen Fonds sowohl regulative wie distributive Instrumente supranationaler europäischer Solidarität mit unterschiedlichen Adressaten. Im regulativen Sinne existiert eine Reihe primär- und sekundärrechtlich festgeschriebener Mindeststandards sowie koordinierender Flankierungen, wie der „Soziale Dialog“ (Art. 138 EGV) oder die neu im Vertrag von Lissabon hinzugefügte Sozialklausel (Art. 9 AEUV). Empfänger supranational koordinierter distributiver Solidarakte sind einzelne soziale Gruppen, wie etwa Landwirte (Agrarfonds) oder verschiedene Arbeitnehmergruppen (Europäischer Sozialfonds), und Regionen (Kohäsionsfonds), aber auch Staaten, beispielsweise Beitrittskandidaten (PHARE, ISPA, SAPARD, IPA). ${ }^{9}$

Eine intergouvernementale Solidarität impliziert eine Hilfsbereitschaft von Regierungen als kollektive Volksvertreter untereinander. Angesichts des primär zwischenstaatlichen Entscheidungsverfahrens bei Erweiterungen der EG bzw. EU kann hier auch der Appell der Beitrittskandidaten an eine europäische Solidarität eingeordnet werden. In diesem Sinne wurde spätestens mit der Süderweiterung der EG appelliert, sich nicht als „Klub der Reichen“ weniger entwickelten Beitrittsaspiranten zu versperren (vgl. Garrigues 1977; Contogeorgis 1978). Ähnlich wurde im Zuge der Osterweiterung der EU vor einem neuen „Jalta“ gewarnt, das hinter einem „ökonomischen Eisernen Vorhang“ die östliche Seite in Armut verharren lässt (Saryusz-Wolski 1994, S. 20-21; siehe auch Stawarska 1999). Diese intergouvernementale Solidarität ist auch ein regelmäßig wiederkehrendes Moment in den Verhandlungen zur Finanziellen Vorausschau. Während die Schaf-

9 Im Folgenden berücksichtigen wir auch Beitrittskandidaten, da bisher nur im britischen (1963) und norwegischen Fall (1972 und 1994) das Beitrittsverfahren nicht erfolgreich abgeschlossen werden konnte. Zudem war bereits im Gründungsmythos in den 1950er-Jahren die paneuropäische Ausrichtung der europäischen Integration angelegt. 
fung, die Bestimmung der finanziellen Ausstattung und der Zweck von Gemeinschaftsfonds der intergouvernementalen Solidarität zugeordnet werden kann, ist die konkrete Verteilung an die Adressaten supranational organisiert.

Die Eurokrise wirft die Frage nach solidarischem Handeln auf allen vier Ebenen auf. Während die wachsenden sozialen Ungleichheiten innerhalb der Krisenstaaten Forderungen nach einer stärkeren intranationalen Solidarität provozieren, stellen die ersten grenzüberschreitenden Protest- und Unterstützungsaktionen sowie vereinzelte Forderungen nach einem stärkeren sozialen Europa Elemente einer transnationalen bzw. supranationalen Solidarität dar. Diese drei Konzeptionen europäischer Solidarität blenden wir im Folgenden jedoch aus, da im Zentrum der Debatte bisher primär eine Solidarität zwischen den Mitgliedstaaten stand, die in Form von spontan geschnürten Rettungspaketen und mittlerweile auch längerfristig angelegten Institutionen den Krisenstaaten auf Regierungskonferenzen gewährt wurde. ${ }^{10}$ Wir entwickeln nun drei idealtypische Modelle einer intergouvernementalen europäischen Solidarität, die sich insbesondere hinsichtlich der beiden zuerst genannten Dimensionen - Motivation der solidarisch Handelnden und Ausrichtung der Solidarleistungen - unterscheiden.

Tabelle 1: Dimensionen europäischer Solidarität

\begin{tabular}{|c|c|c|}
\hline $\begin{array}{c}\text { Motivation } \\
\text { der solidarisch Handelnden }\end{array}$ & $\begin{array}{c}\text { Ausrichtung } \\
\text { der Solidarleistungen }\end{array}$ & Akteursebene \\
\hline $\begin{array}{c}\text { gemeinwohlorientiert } \\
\text { eigennutzorientiert }\end{array}$ & $\begin{array}{c}\text { horizontal } \\
\text { vertikal }\end{array}$ & $\begin{array}{c}\text { intranational } \\
\text { transnational } \\
\text { supranational } \\
\text { intergouvernemental }\end{array}$ \\
\hline
\end{tabular}

Quelle: eigene Darstellung

\subsection{Drei idealtypische Modelle einer intergouvernementalen europäischen Solidarität}

Eine zwischenstaatliche Hilfsbereitschaft kann als föderative, organische oder distributive Solidarität konzeptionalisiert werden. Im Folgenden werden diese drei Idealtypen im Weberschen Sinne „durch einseitige Steigerung eines oder einiger Gesichtspunkte [...] vorhandener Einzelerscheinungen [...] zu einem in sich einheitlichen Gedankengebilde“ (Weber 1951 [1904], S. 190-191) entwickelt. Zur präzisen Klärung von Konzepten erscheint eine Idealtypenbildung gerade dann sinnvoll, wenn - wie im Falle der europäischen Solidarität - unterschiedliche Begriffsverständnisse hinsichtlich mehrerer Merkmale erheblich variieren (Stinchcombe 1968, S. 43-45). Ein Idealtyp ist als „idealer Grenzbegriff“ (Weber 1951 [1904], S. 194) eine explizit kontrafaktische Überzeichnung der zentralen, „genetischen" Merkmale eines Phänomens und bildet dadurch einen Pol auf einem Kontinuum dieser Merkmalsausprägungen (Hendricks u. Peters 1973; Lindbekk

10 Insbesondere die sich zuspitzende Frage transnationaler Solidarität gewinnt derzeit an Brisanz. Eine ausführliche Beschäftigung erscheint jedoch an dieser Stelle zu komplex und würde einen weiteren Aufsatz erfordern. 
1992, S. 290). In einem deskriptiv-komparativen Verständnis stellen Idealtypen statische Vergleichskategorien dar, an denen die empirisch beobachtbaren heterogenen Einzelerscheinungen gemessen werden können. ${ }^{11}$ Dieses deduktive Vorgehen unterscheidet sich somit von einer induktiven Typenbildung über empiriegeleitete Clusterbildung (siehe Kluge 1999, S. 51-85) oder über eine ideengeschichtliche Rekonstruktion konkurrierender Solidaritätskonzeptionen.

Die entlang der oben erläuterten Merkmale „Motivation der solidarisch Handelnden“ und „Ausrichtung der Solidarleistungen“ entstehenden Idealtypen (Abbildung 1) werden zur Illustration und theoretischen Einbettung an einflussreiche politische Theorien geknüpft. ${ }^{12}$ Wenngleich diese Idealtypen mit sehr unterschiedlichen Solidarbedingungen und -leistungen, verschiedenem Umfang und Häufigkeit von Solidarakten sowie bezogen auf die Europäische Union mit alternativen Finalitätsvorstellungen des europäischen Integrationsprozesses verbunden sind, sind diese Modelle komplementär, da sie gleichzeitig auftreten können.

\section{Abbildung 1: Drei Idealtypen intergouvernementaler europäischer Solidarität}

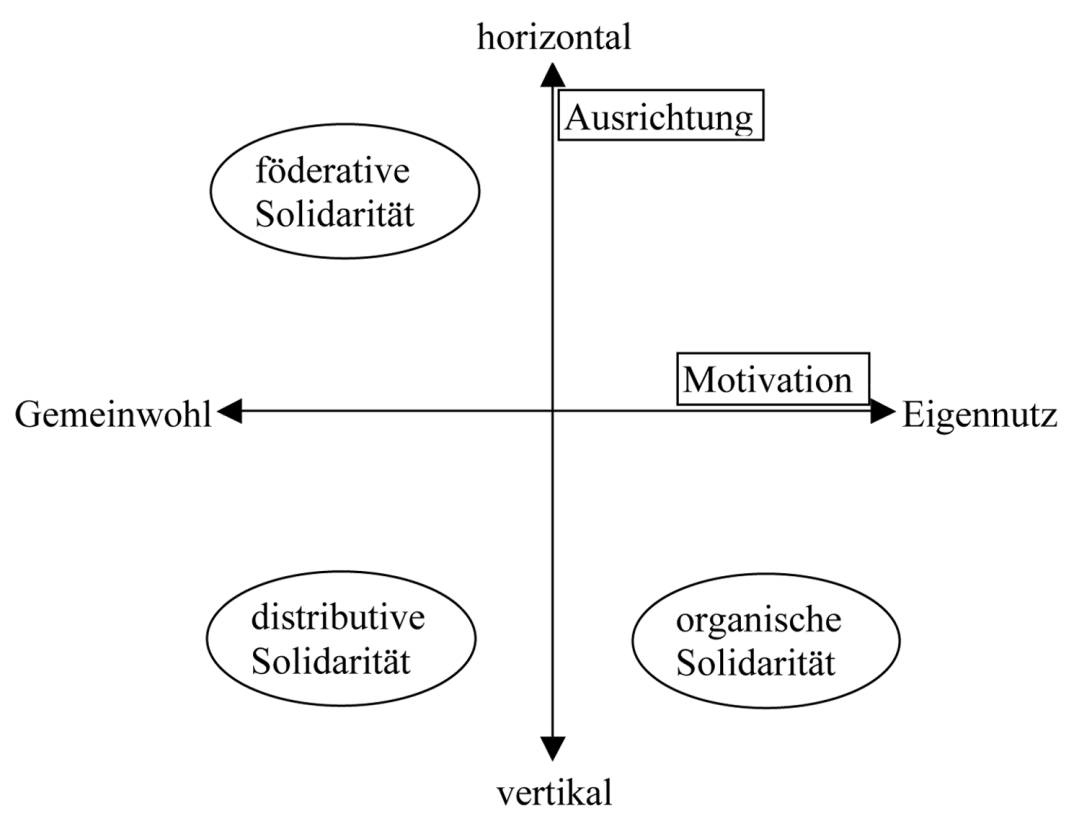

Quelle: eigene Darstellung

11 Damit wird kein explanativer Anspruch erhoben und die generische Entwicklung sowie die sozialen Entstehungsbedingungen von Solidarität werden ausgeblendet. Zur Kritik an dem sowohl deskriptiven als auch explanativen Anspruch bei Weber siehe Hendricks u. Peters 1973; Papineau 1976. Zu den Entstehungsbedingungen von Solidarität und kollektiver Identität im Rahmen der Nationalismusforschung siehe etwa Deutsch 1953; Anderson 1983.

12 Illustrativ sind diese ideengeschichtlichen Rekurse in dem Sinne, dass eine vollkommene Kongruenz der Idealtypen mit der jeweiligen politischen Theorie nicht unterstellt wird. Für ein vergleichbares Vorgehen siehe Wendt (1999, S. 246-312) und Karolewski (2005). 
Das Modell der föderativen Solidarität ist von Immanuel Kants Schrift „Zum Ewigen Frieden“ (1984 [1795]) inspiriert. Sein souveränistischer Zugang unterstellt eine friedliche Koexistenz autonom agierender Demokratien, die sich in einem „Föderalism freier Staaten“ zur Schaffung „eines den Krieg abwehrenden, bestehenden und sich immer ausbreitenden Bundes" (Kant [1795] 1984, S. 20, Herv. im Original) zusammenschließen. ${ }^{13}$ Wenngleich Kant in der pazifizierenden Kraft demokratischer Gesellschaften den Schlüssel zu einem ewigen Frieden sah, impliziert bereits der Begriff des Bundes („foedus“) eine gegenseitige Beistandsverpflichtung, die über einen schlichten Nichtangriffspakt deutlich hinausgeht. Der Bundesbegriff, für den Kants Friedensschrift und sein Ausdruck des Völkerbundes wegweisend war, enthält eine Betonung von Gefühlsmomenten und ist ideengeschichtlich schon seit dem frühen Mittelalter eng mit dem Begriff der Bruderschaft („fraternitas“) verbunden (Koselleck 1979, S. 582 ff.), wobei die Bundesmitglieder sowohl eine strukturelle Gemeinsamkeit als auch durch gegenseitige Hilfe in einem Kampf nach außen geeint sind (Koselleck 1979, S. 599 u. 636). ${ }^{14}$ In seiner späten Schrift „The Law of Peoples“ folgt auch John Rawls dieser Konzeption, gewährt aber nun explizit ein Kriegsrecht zur Verteidigung von Alliierten (1999, S. 91) und öffnet so das Bündnis auch für illiberale, aber achtbare Staaten. ${ }^{15}$ So verstanden wird eine föderative Solidarität nur in Extremsituationen existenzieller äußerer Bedrohung eingefordert, sodass Solidarleistungen selten und im Umfang lediglich auf die Verteidigung des Status quo begrenzt sind. ${ }^{16}$

Eine föderative europäische Solidarität ist vereinbar mit der flachen Integrationslogik eines europäischen Staatenbundes im Sinne von De Gaulles „Europa der Vaterländer“. Aus dieser Perspektive fungieren die konkurrierenden globalen Gravitationszentren als einigender Kontrapunkt. Trotz des Aufbaus militärischer Kapazitäten im Rahmen der Europäischen Sicherheits- und Verteidigungspolitik scheint eine echte Verteidigungsunion auf absehbare Zeit ebenso wenig möglich wie eine Verteidigung in einem konventionellen Krieg nötig. Gleichwohl gibt es Formen föderativer empirischer Solidarität, wie im Rahmen einer polizeilichen Kooperation zur Bekämpfung von Kriminalität und Terrorismus (Europol). Im Bereich der Energiepolitik wird eine Solidarität („Energiesolidarität“) angesichts der Abhängigkeit einiger Staaten von russischen Energieträgern angemahnt (Baumann 2008, Geden 2007, kritisch dazu Götz 2009). Im Konflikt mit Russland um

13 Wenngleich Kant der demokratischen Regierungsform kritisch gegenüberstand und von Republiken sprach, finden letztere bekanntlich in modernen Demokratien durchaus ihren Ausdruck.

14 Hier werden Parallelen zur Kampf-Solidarität der französischen Revolution und deutschen Arbeiterbewegung (Bayertz 1998b), vgl. Fußnote 5, sowie zu Durkheims mechanischer Solidarität ([1893] 1977, S. 118-161) deutlich.

15 Rawls fordert auch die Unterstützung „, belasteter Völker“, denen eine faire Institutionenordnung aufgrund von Ressourcenarmut verwehrt bleibt. Der hier entwickelte Begriff der föderativen Solidarität ist jedoch ohne Verteilungsauswirkungen zu verstehen, zumal das Existenzminimum für eine wohlgeordnete Gesellschaft innerhalb der EU bereits gesichert ist.

16 Es sei angemerkt, dass trotz der impliziten Beistandsverpflichtung innerhalb eines Bundes weder Kant noch Rawls in diesem internationalen Kontext explizit von „Solidarität“ sprechen. John Rawls verwendet zwar nicht den Begriff der Solidarität, bezieht sich aber auf eine „natürliche Bedeutung der Brüderlichkeit“, die sich ausdrücklich im Unterschiedsprinzip manifestiert (Rawls [1971] 1975, S. 126-127). Zur semantischen Unterscheidung der beiden Begriffe siehe Bayertz 1998b, S. 15-16. 
den Export polnischen Fleisches lobte beispielsweise Warschau die Solidarität, die es seitens anderer EU-Mitgliedstaaten erfuhr (Marek 2010, S. 39).

Das Modell der organischen Solidarität orientiert sich an Émile Durkheim ([1893] 1977). Sein zentraler Gedanke lautet, dass Solidarität als ein die Gesellschaft zusammenhaltendes Fundament keinesfalls eine Gleichartigkeit ihrer Mitglieder erfordert. Im Gegenteil, aufgrund der arbeitsteiligen Interdependenz von unterschiedlich spezialisierten Gesellschaftsmitgliedern sind die Individuen nicht mehr beliebig austauschbar, wodurch das sie verbindende funktionalistische Band beständiger und belastbarer ist als in primitiven, nicht differenzierten Gesellschaften. Analog zu den einzelnen Organen bei höher entwickelten Tieren „ist die Einheit des Organismus umso größer, je stärker die Individualisierung der Teile ausgeprägt ist“ (Durkheim [1893] 1977, S. 183). Da Durkheim die Gesellschaft endogen betrachtet und externe Schocks ausblendet, ist die organische Solidarität vertikal ausgerichtet. Aus einer individuellen handlungstheoretischen Perspektive gesehen sind die rationalen Akteure prinzipiell bereit, distributive Maßnahmen zur Unterstützung notleidender Gemeinschaftsmitglieder solange hinzunehmen, wie sie die zentralen sozialen Güter nur innerhalb dieses Kooperationsgefüges erwerben können (vgl. Mau 2009, S. 68). Solidarleistungen werden in der Regel sporadisch, etwa in Krisenzeiten, gewährt und richten sich in ihrem Umfang an einem Minimum aus, das für die Aufrechterhaltung der Funktionsfähigkeit der betroffenen Individuen nötig ist. Das sich daraus entwickelnde Kollektivbewusstsein bedarf daher keiner republikanischen Gemeinwohlorientierung des individuellen Handelns oder gemeinsamer ethnischer Wurzeln, sondern ist durchaus vereinbar mit einem primär eigennützigen Handlungskalkül ziviler Bürger (Baum 1975; Baurmann 1998, S. 346-347; Mau 2009, S. 67-71). ${ }^{17}$ Somit bildet die organische Solidarität ein nicht-intendiertes Desiderat eines sich spezialisierenden und stetig integrierenden Kooperationsgefüges, dessen Intensität und Reichweite mit der Interaktionsdichte und funktionalen Differenzierung der Gesellschaftsmitglieder zunimmt. Damit wird aber auch ein Ausschluss von Gesellschaftsmitgliedern möglich: Sobald die Kosten der Unterstützung notleidender Gesellschaftsmitglieder die Kooperationsgewinne übersteigen, wird ein Ausscheiden aus dem Kooperationsverbund wahrscheinlich. Damit stellt Solidarität in diesem Verständnis keinen mobilisierenden „moralischen Kampfbegriff“ (Imbusch u. Rucht 2005, S. 24) wie im föderativen Modell dar, noch ist sie Ausdruck von Nächstenliebe, sondern sie ist eine sich aus der allseitig nützlichen Arbeitsteilung ergebende Bindekraft.

Die Voraussetzungen für eine organische europäische Solidarität scheinen angesichts der bislang vorrangig negativen Integration der europäischen Volkswirt-

17 Dies ist ohne Zweifel eine Zuspitzung des Durkheimschen Solidaritätskonzepts, denn Durkheim selbst unterscheidet durchaus zwischen einem negativ konnotierten Egoismus und einem positiv eingefärbten Individualismus (vgl. Durkheim 1893 [1992], S. 468; auch Fournier 2005, S. 54); Solidarität und Moral verwendet er kongruent (Luhmann 1992, S. 24). Gleichwohl kann eingewendet werden, dass zum einen die Austauschbeziehungen auch aus nutzenmaximierenden Motiven entstehen können (Müller und Schmid 1992, S. 514-515) und zum anderen Durkheim nur unzureichend erläutert, wie aus wechselseitiger Interdependenz eine moralische Handlungsdisposition entstehen soll (Schmid 1989, S. 528). Letztlich erscheint die bei ihm als organisch bezeichnete Solidarität durchaus mit einem egoistischen Verhaltensmodus vereinbar. 
schaften und der damit einhergehenden ökonomischen Interdependenz sowie der relativ schwach ausgeprägten europäischen Identität erfüllt zu sein (vgl. Streeck 2000, S. 256). ${ }^{18}$ Die Infrastrukturfinanzierungen des Kohäsionsfonds können beispielsweise in diesem organischen Sinne gedeutet werden, da sie die Transaktionskosten eines grenzüberschreitenden und arbeitsteiligen Produktionsprozesses und Vertriebs reduzieren. Aus einer sicherheitspolitischen Perspektive kann selbst der Agrarfonds als Gewährleistung von Autarkie interpretiert werden, wenngleich dies kaum das Motiv für seine Schaffung und Aufrechterhaltung war.

Das Modell der distributiven Solidarität basiert auf dem egalitär-liberalen Gerechtigkeitsverständnis von John Rawls (1975 [1971]). Die gegenseitige Hilfsbereitschaft geht deutlich über die sporadische Bekämpfung externer Bedrohungen zur Verteidigung der negativen Freiheit von Staaten oder eine bloße Gewährleistung der Funktionsfähigkeit der Mitglieder eines Kooperationsverbundes zugunsten einer allseitig nutzenmaximierenden Wohlstandssteigerung hinaus. ${ }^{19}$ In seiner „Theorie der Gerechtigkeit“ plädierte Rawls für ein Unterschiedsprinzip, wonach eine Ungleichheit von sozialen Gütern nur dann als fair gilt, wenn sie den am wenigsten Begünstigten besser stellt (Rawls 1975 [1971], S. 96). Zwei Rechtfertigungsstränge lassen sich bei ihm erkennen (vgl. Kymlicka 1990): Das intuitive Argument lautet, dass die natürliche Ungleichverteilung der individuellen Ausstattungen mit angeborenen Talenten, Eigenschaften oder Fähigkeiten als Ergebnis der willkürlichen Lotterie der Natur deswegen durch soziale Institutionen ausgeglichen werden muss, da Ansprüche prinzipiell auf Entscheidungen und nicht auf derartig zufälligen Handlungsbedingungen beruhen dürfen. Das kontraktualistische Argument unterstellt hingegen einen fiktiven Urzustand, in dem die rationalen Wähler aufgrund eines Schleiers des Unwissens keine Informationen über ihre persönlichen Eigenschaften und sozialen Positionen besitzen und aufgrund der Verbindlichkeit ihrer einmaligen Wahl risikoavers einer Gesellschaftsordnung zustimmen würden, welche die schlechtestmögliche Position in der Gesellschaft maximiert. Aus der Rawlsschen Gerechtigkeitstheorie lässt sich mithin ein vertikal ausgerichtetes Solidaritätsverständnis ableiten, wonach zufällige Ungleichverteilungen zwischen den Mitmenschen kompensiert werden sollten. Dies führt zu einem permanenten Umverteilungsmechanismus mit erheblichen distributiven Konsequenzen. Eine entscheidungs-, aber nicht ausstattungsabhängige (vgl. Dworkin 1981, S. 311) Solidarität hat jedoch dort ihre Grenzen, wo die Eigenverantwortung beginnt: Je stärker die Notlage selbst verschuldet ist, desto geringer wird die Hilfsbereitschaft des Bessergestellten. ${ }^{20}$

Allerdings weist Rawls selbst darauf hin, dass seine Gerechtigkeitstheorie auf den (wohlgeordneten) Nationalstaat beschränkt bleibt, da es an einem geteilten

18 Streecks Plädoyer für eine „competitive solidarity“ kann als die normative Wendung dieses Konzepts betrachtet werden. Während ihm zufolge eine innerstaatliche Kohäsion durch eine mechanische Solidarität gewährleistet werden kann, sollten die externen Beziehungen innerhalb der EU einer organischen Solidarität überlassen werden (vgl. Streeck 2000, S. 260).

19 Dieses Konzept ähnelt Streecks (2000, S. 246) „,redistributive solidarity“, das er allerdings explizit auf die innerstaatliche Dimension beschränkt.

20 Diese Kritik an seinem ursprünglichen Unterschiedsprinzip hat Rawls später (1999, S. 108) akzeptiert. 
Gerechtigkeitssinn auf internationaler Ebene mangele (Rawls [1971] 1975, S. 498; Waldron 1987, S. 145). Insofern ist eine Übertragung dieses vertikalen Solidaritätskonzepts auf die Europäische Union zumindest gewagt, denn es existiert kaum eine belastbare kollektive Identität (Deutsch 2005, S. 171), die „unkompensierte Sonderopfer" legitimieren würde (Scharpf 2002). Gleichwohl lassen sich zwei Argumente dafür anführen, diesen Solidaritätsbegriff dennoch auf die Europäische Union anzuwenden: Erstens gleicht sie immer weniger einer internationalen Organisation, da sie mittlerweile einige klassische Merkmale eines europäischen Bundesstaates aufweist, wie z. B. den Vorrang des europäischen vor dem nationalen Recht oder die Wirtschafts- und Währungsunion. Außerdem besteht ein beachtlicher Wertekonsens, der sich in der Europäischen Grundrechtecharta und den Kopenhagener Kriterien manifestiert, sodass durchaus von einem grenzüberschreitenden kollektiven Gerechtigkeitssinn gesprochen werden kann. Zweitens kann mit Charles Beitz auch der Einsatz eines globalen Schleiers des Unwissens - analog zu den erwähnten moralischen Rechtfertigungsgründen für ein solidarisches Handeln - damit gerechtfertigt werden, dass einerseits natürliche Ressourcen als Bedingung für eine erfolgreiche ökonomische Entwicklung willkürlich verteilt sind und andererseits moderne Staaten nicht autark agieren, sondern voneinander abhängig sind, woraus soziale Verpflichtungen und die Notwendigkeit fairer Kooperationsregeln resultieren (Beitz 1979, S. 141-142). Daher fordert Beitz im Gegensatz zu Rawls ,an international difference principle [...] in the sense that it is the globally least advantaged representative person (or group of persons) whose position is to be maximized " (Beitz 1979, S. 152). ${ }^{21}$ Während sein erstes Argument empirisch mit dem Hinweis darauf relativiert werden kann, dass Rohstoffe nur bedingt die wirtschaftliche Entwicklung eines Staates beeinflussen - wie Japans Rohstoffarmut und Argentiniens Reichtum an natürlichen Ressourcen verdeutlichen (Rawls 1999, S. 108) - und erst recht unter den EUMitgliedstaaten wenig ausschlaggebend sein dürfte, spricht die fortschreitende Globalisierung im Allgemeinen und die wirtschaftliche Verflechtung innerhalb der EU im Besonderen durchaus für eine Übertragung der Rawlsschen Gerechtigkeitskonzeption auf eine zwischenstaatliche Ebene.

In einem solchen distributiven Sinne existiert eine europäische Solidarität allenfalls in Ansätzen. Es nimmt wenig Wunder, dass gerade diejenigen Politikbereiche mit hohen finanziellen Kosten und redistributiven Konsequenzen, wie Arbeitsund Sozialpolitik, nicht integriert wurden. Dennoch existiert seit den Römischen Verträgen mit dem Europäischen Sozialfonds ein Instrument redistributiver europäischer Sozialpolitik, das finanzielle Mittel (ca. 10 Milliarden Euro) zur Prävention und Bekämpfung von Arbeitslosigkeit, beruflicher Qualifikation, sozialer Integration in den Arbeitsmarkt sowie Gleichstellung von Frau und Mann bereitstellt. Zudem wurde 2006 ein Globalisierungsfonds zur Kompensation von entlassenen Angestellten gegründet, die aufgrund der globalen Standortkonkurrenz - also unverschuldet - ihre Arbeit verloren haben. Der Artikel 222 (AEUV), wonach im Falle eines Terroranschlags, einer Naturkatastrophe oder einer von

21 Vgl. die Gegenposition bei Rawls 1999, S. 166ff. 
Menschen verursachten Katastrophe Solidarität mit allen verfügbaren Mitteln angemahnt wird, kann je nach Funktionsbeeinträchtigung sowohl der organischen als auch der distributiven Solidarität zugeordnet werden.

Tabelle 2: Drei Idealtypen intergouvernementaler europäischer Solidarität

\begin{tabular}{|l|c|c|c|}
\hline Solidaritätsmodell & föderativ & organisch & distributiv \\
\hline Merkmale & $\begin{array}{c}\text { gemeinwohlorientiert } \\
\text { (Bündnistreue) }\end{array}$ & $\begin{array}{c}\text { eigennutzorientiert } \\
\text { (Verflechtung) }\end{array}$ & $\begin{array}{c}\text { gemeinwohlorientiert } \\
\text { (Fairness) }\end{array}$ \\
\hline Ausrichtung & horizontal & vertikal & vertikal \\
\hline $\begin{array}{l}\text { Zweck der } \\
\text { Solidarleistungen }\end{array}$ & $\begin{array}{c}\text { Abwehr zur Wahrung } \\
\text { des Status quo }\end{array}$ & $\begin{array}{c}\text { Minimalunterstützung } \\
\text { zur Wiederherstellung } \\
\text { des Status quo }\end{array}$ & $\begin{array}{c}\text { Rekompensation für } \\
\text { willkürliche Hand- } \\
\text { lungseinschränkungen }\end{array}$ \\
\hline Solidarbedingungen & $\begin{array}{c}\text { exogene existenzielle } \\
\text { Bedrohung }\end{array}$ & $\begin{array}{c}\text { kritische Funktions- } \\
\text { einschränkung eines } \\
\text { elementaren } \\
\text { Gesellschaftsmitglieds }\end{array}$ & $\begin{array}{c}\text { unverschuldete } \\
\text { Schlechterstellung } \\
\text { eines Gesellschafts- } \\
\text { mitgliedes }\end{array}$ \\
\hline Umfang & $\begin{array}{c}\text { gering } \\
\text { (Verteidigung) }\end{array}$ & $\begin{array}{c}\text { mittel } \\
\text { (Funktionssicherung) }\end{array}$ & $\begin{array}{c}\text { hoch } \\
\text { (Ausgleichszahlungen) }\end{array}$ \\
\hline Häufigkeit & selten & sporadisch & permanent \\
\hline $\begin{array}{l}\text { Anwendbarkeit auf } \\
\text { die EU (AEUV) }\end{array}$ & Europol (Art. 88) & $\begin{array}{c}\text { Kohäsionsfonds } \\
\text { (Art. 177) }\end{array}$ & $\begin{array}{c}\text { Europäischer Sozial- } \\
\text { fonds (Art. 162) }\end{array}$ \\
\hline Finalitätsvorstellung & Staatenbund & Wirtschaftsverbund & Bundesstaat \\
\hline
\end{tabular}

Quelle: eigene Darstellung

\section{Intergouvernementale europäische Solidarität in Zeiten der Eurokrise}

Durch dieses konzeptionelle Prisma kann nun das Verhalten der Mitgliedstaaten in der Eurokrise dahingehend analysiert werden, in welchem Sinne Solidarität eingefordert und geleistet wurde.

Aufgrund der eingeschränkten Zahlungsfähigkeit von Griechenland (ab Mai 2010), Irland (ab November 2010) und Portugal (ab April 2011) entschied sich der Rat diesen Eurostaaten gemeinsam mit dem IWF sukzessive Kredite zur Abwendung von Staatsinsolvenzen zu gewähren. Neben einem einmaligen Direktkredit von 110 Milliarden Euro an Griechenland wurden zum Zweck einer Eindämmung der Krise erst ein Europäischer Finanzstabilisierungsmechanismus (EFSM) mit einem Volumen von 750 Milliarden Euro (Mai 2010) und später ein permanent angelegter Europäischer Stabilitätsmechanismus (ESM) als eigenständige internationale Finanzinstitution mit einer Kapazität von 500 Milliarden Euro (September 2012) geschaffen. Darüber hinaus kaufte die Europäische Zentralbank parallel Anleihen von Krisenländern an und gewährte günstige Kredite für Banken, während im Falle Griechenlands private Gläubiger einem partiellen Schuldenschnitt zustimmten (März 2012). Nach wie vor ist allerdings kein Ende der Eurokrise absehbar, denn nachdem auch Spanien und Zypern im Juni 2012 
finanzielle Unterstützung aus dem EFSF beantragt haben, gelten auch Slowenien und Italien weiterhin als bedroht. ${ }^{22}$

Die öffentliche Debatte innerhalb der EU oszilliert gegenwärtig zwischen erbetener Solidarität seitens der Geberstaaten und eingeforderter Solidität in der Haushaltspolitik der Krisenstaaten - und beides steht zur Disposition: Einerseits deutet der gescheiterte Versuch Finnlands, für seine Kredite an Griechenland auf Garantien zu bestehen, die schleichende Erosion der Hilfsbereitschaft der wohlhabenderen Staaten an. ${ }^{23}$ Andererseits gab es wiederholt Kritik an der mangelnden Umsetzung von Reformankündigungen insbesondere in Griechenland, aber auch in Italien (vgl. European Commission et al. 2011). Damit steht die Reichweite der Solidarleistungen und ihrer Bedingungen im Mittelpunkt, doch diese hängen wiederum vom konkreten Solidaritätsverständnis ab.

Zur Mobilisierung der Opferbereitschaft in den Geberstaaten wurde vielerorts auf eine Solidarität im föderativen Sinne rekurriert: So zeichneten zahlreiche prominente Mitglieder der Bundesregierung, aber auch führende Vertreter der EU das Bild einer in den stürmischen Gewässern der Globalisierung gefährdeten Gemeinschaft, die im 21. Jahrhundert mit anderen globalen Machtzentren sowohl politisch als auch ökonomisch nur gemeinsam konkurrieren könne. ${ }^{24}$ In dieses Bild fügt sich das Verbot von ungedeckten Leerverkäufen, die unter anderem die unkontrollierten und destabilisierenden Aktivitäten von Spekulanten domestizieren sollen, wobei die partielle Einführung einer Finanztransaktionssteuer in nur 11 EU-Mitgliedstaaten im Rahmen einer verstärkten Kooperation die Grenzen einer solchen Solidarität illustriert. Auch die Eigenständigkeit der drei führenden USamerikanischen Ratingagenturen Standard \& Poor‘s, Moody‘s und Fitch wurde in diesem Sinne kritisiert (Bundesfinanzministerium 2011), da deren mitunter überraschende Herabstufungen der Kreditwürdigkeit von strauchelnden Staaten in eine gefährliche Abwärtsspirale aus Überschuldung und sinkender Bonität zu münden drohen. Der Plan, eine eigene europäische Ratingagentur zu gründen (EUobserver 2010), scheiterte jedoch an mangelndem Interesse von Investoren.

Die ersten substanziellen Rettungsversuche und die Intervention der Europäischen Zentralbank lassen sich hingegen einer organischen Solidarität zuordnen, denn einerseits hätte eine Umschuldung im Falle eines einzukalkulierenden Zahlungsausfalls faktisch redistributive Effekte und andererseits orientierten sich die finanziellen Garantien am Minimum, das für die Funktionsfähigkeit der bedrohten Volkswirtschaften notwendig erschien. Der sukzessive Ausbau des Rettungssystems war dabei offensichtlich nur Ausdruck einer Korrektur dessen, was zum gegebenen Zeitpunkt als insolvenzverhindernde conditio sine qua non wahrgenommen wurde. Der Diskurs spiegelte die Handlungslogik einer organischen Solidarität deutlich wider. Ex negativo wurde auf die Alternativlosigkeit der Ret-

22 Für eine ausführliche Darstellung des Krisenverlaufs siehe beispielsweise Berger u. Ücker (2011).

23 Siehe dazu die offene und umstrittene Formulierung des Punktes 9 in Council of the European Union 2011, S. 3.

24 Vgl. den auf Initiative des Bundesumweltministers Röttgen gefassten Beschluss des CDU-Landesvorstands Nordrhein-Westfalen (2011) sowie die Positionen der Bundesminister von der Leyen (ZEIT ONLINE, 2011a) und Schäuble (WirtschaftsWoche Online, 2011) sowie Bundeskanzlerin Merkel (Tagesspiegel Online, 2011) sowie Barroso 2012, S. 5-6, und Pöttering 2012, S. 14. 
tungsversuche in einer bezeichnenden und inflationär verwendeten Metapher hingewiesen: Ein Ausscheiden Griechenlands aus dem Euroraum würde eine immanente ,Ansteckungsgefahr' für andere bedrohte Staaten bergen. ${ }^{25}$ Positiv gewendet erinnerten die Befürworter der Rettungsaktionen hingegen daran, dass gerade die Geberstaaten - allen voran Deutschland - von dem gemeinsamen Binnenmarkt profitierten (vgl. z. B. Bundesregierung 2007).

Im weiteren Krisenverlauf deuten allerdings einige Anzeichen darauf hin, dass die Eindämmungsversuche der Mitgliedstaaten allmählich über eine organische Solidarität hinausgehen und sich zunehmend an einer distributiven Solidarität orientieren. Immerhin stehen die Kosten der inkrementalen Schuldenvergemeinschaftung für die Geberstaaten in zunehmendem Missverhältnis zum Nutzen an der Teilhabe der Krisenstaaten am gemeinsamen Binnenmarkt. ${ }^{26}$ Einerseits erstrecken sich die Solidarleistungen mittlerweile auf einen EFSM, der sogar präventiv Staatsanleihen von Krisenstaaten erwerben kann und somit de facto eine Haftungsgemeinschaft zementiert, da letztlich die Geberstaaten in der Eurozone - direkt oder indirekt - im Bedarfsfall für die Verpflichtungen größtenteils aufkommen müssen. Andererseits wurden die Solidarbedingungen nun immer mehr betont. In Form von pro-aktiven Präventionsmaßnahmen zielen das „Six-Pack“ und der Europäische Fiskalpakt auf eine Kontroll- und Sanktionsverschärfung der mitgliedstaatlichen Haushaltspolitik. ${ }^{27}$ Das im September 2011 beschlossene Maßnahmenprogramm („Six-Pack“) umfasst eine Verschärfung des Stabilitätspakts, härtere halbautomatische Sanktionsmaßnahmen, raschere Schuldenreduzierung, strengere Ausgabenkontrolle, eine verfassungsmäßig verankerte Schuldenbremse sowie von der EU-Kommission vorgeschriebene Maßnahmen zur Steigerung der Wettbewerbsfähigkeit. Der von allen EU-Mitgliedstaaten außer Großbritannien und Tschechien unterschriebene und voraussichtlich im Januar 2013 in Kraft tretende Europäische Fiskalpakt konkretisiert und verschärft die Regeln nationaler Schuldenpolitik. Auch die jüngst diskutierte und in Teilen vereinbarte Bankenunion kann letztlich der distributiven Solidarität zugerechnet werden. ${ }^{28}$ Die im Dezember 2012 von den Staats- und Regierungschefs der EUMitgliedstaaten beschlossene Bankenaufsicht systemrelevanter Banken („too big to fail“) entspricht einem entscheidungsabhängigen Gerechtigkeitsverständnis. Aus dieser Perspektive erscheint es unfair, wenn private Banken bei spekulativen Geschäften Gewinne für sich verbuchen können, aber im Verlustfall zur Vermei-

25 Vgl. die Äußerungen des damaligen Direktors des Internationalen Währungsfonds Dominique Strauss-Kahn in ZEIT ONLINE (2010), des Vorsitzenden der Eurozone Jean-Claude Juncker in WirtschaftsBlatt (2011) sowie des damaligen Präsidenten der Europäischen Zentralbank JeanClaude Trichet in SPIEGEL ONLINE (2010).

26 Vergleicht man aus deutscher Perspektive den Durchschnitt der jährlichen Überschüsse in der deutschen Handelsbilanz zwischen 2000 und 2009 von 140,29 Milliarden Euro (Statistisches Bundesamt) mit dem kontinuierlich wachsenden Gesamtschuldenvolumen allein von Griechenland von rund 329 Milliarden Euro (Eurostat) oder der Haftungsobergrenze für Deutschland von 211 Milliarden im Rahmen des ESM, so deutet sich zumindest ein sich verschärfendes Ungleichgewicht an. Gleichwohl ist eine exakte Kosten-Nutzen-Rechnung nur schwer möglich, da zahlreiche Faktoren bei dieser Gegenüberstellung ausgeblendet werden.

27 Zur Unterscheidung der reaktiven und pro-aktiven Maßnahmen siehe ausführlicher Beichelt u. von Ondarza 2011.

28 Großbritannien und Schweden haben sich für ein Opt-out entschieden. 
dung von Systeminstabilität über eine Staatsintervention indirekt auf Kosten der nationalen Steuerzahler gerettet werden müssen. Im Falle Irlands führte eine solche alternativlose Stützung nationaler Banken zu einer drohenden Staatsinsolvenz, die nur mit externer Hilfe verhindert werden konnte. ${ }^{29}$ Als Ausdruck des Prinzips der Eigenverantwortung können aber auch sowohl die von der Europäischen Kommission vorgeschlagene EU-weite permanente Bankenabgabe als auch die Beteiligung privater Gläubiger in Höhe von 50 Milliarden Euro bis 2014 im griechischen Fall verstanden werden. Als Sanktionsmaßnahmen wurden bereits die Aussetzung der Zuteilungen der nationalen Kontingente aus den Agrar- und Strukturfonds (Rehn 2010, S. 3), ein nur konditionierter Zugang zu Eurobonds (Reding 2011) und die von Bundesfinanzminister Schäuble vorgeschlagene vertragliche Einführung eines Ausschlussverfahrens als Ultima Ratio bei Nichtkooperation diskutiert (Deutscher Bundestag 2010, S. 2719). ${ }^{30}$ Das Spannungsverhältnis zwischen umfangreichen Solidarleistungen und strengen Solidarbedingungen spiegelt sich auch in der Rhetorik von Bundeskanzlerin Merkel wider, wenn sie einerseits mit ihrem Appell „Scheitert der Euro, dann scheitert Europa!“ (Deutscher Bundestag 2011, S. 15955) die Eurokrise pathetisch zur Herausforderung für eine europäische Schicksalsgemeinschaft stilisiert und andererseits auf Forderungen nach einer Ausweitung der Rettungsmaßnahmen defensiv mit dem Bestehen auf einem ,ausgewogenen Verhältnis zwischen Solidarität und Eigenverantwortung “ (cdu.de 2011) reagiert.

Die bisherigen Maßnahmen können einer distributiven Solidarität in einem weichen Verständnis zugeordnet werden, da weiterhin zwischen nationalen Anleihen mit unterschiedlichen Kreditkonditionen unterschieden wird. Der Vorschlag Jean-Claude Junckers, Gemeinschaftsanleihen (Eurobonds) einzuführen, entspricht hingegen diesem Idealtyp im vollen Sinne, denn diese würden eine ausstattungsunabhängige Chancengleichheit der Eurostaaten am internationalen Finanzmarkt in Form von gleichen Zinsen gewährleisten (siehe Europäische Kommission 2011; Delpla und von Weizsäcker 2011).

Mittlerweile werden aber in der europäischen Debatte zunehmend die konkreten Solidarbedingungen hinterfragt. Denn zum einen scheint eine strenge Austeritätspolitik deswegen kontraproduktiv, da sie konjunkturbremsend wirkt und damit die Zahlungsfähigkeit der betroffenen Staaten weiter einschränkt. Zum anderen wird die unterstellte Selbstverschuldung der Krise seitens der betroffenen Staaten zunehmend angezweifelt, indem insbesondere auf die steigenden Handelsbilanzen Deutschlands verwiesen wird. ${ }^{31}$ Damit ist ein Ende des Solidaritätsdiskurses derzeit nicht absehbar.

29 Die Bankenunion soll zudem einen Abwicklungsfonds für insolvente Banken und eine gemeinsame Einlagesicherung umfassen.

30 Bezüglich der rechtlichen Umsetzbarkeit des Vorschlags gibt es jedoch Zweifel, vgl. Athanassiou 2009, S. 30.

31 Siehe hierzu die Debatte zu den krisenbegünstigenden Effekten der deutschen Außenhandelsüberschüsse, z. B. die damalige französische Finanzministerin und derzeit amtierende IMF-Direktorin Christine Lagarde in FT.com 2010. 


\section{Fazit: Die Risiken einer distributiven europäischen Solidarität}

Angesichts des im Zuge der Eurokrise inflationären, aber uneinheitlichen Rekurses auf eine europäische Solidarität zielte der vorliegende Beitrag auf eine semantische Begriffsbestimmung dieses zentralen Gemeinschaftswerts. Da die Übertragung des Bedeutungskerns auf die Europäische Union mehrere Interpretationen zulässt, wurden entlang der Dimensionen Motivation und Ausrichtung der Solidarakte drei Idealtypen intergouvernementaler europäischer Solidarität - föderativ, organisch und distributiv - entwickelt. Die in der derzeitigen europaweiten Debatte diskutierten und teils auf Regierungskonferenzen beschlossenen Initiativen und Instrumente zur Eindämmung der Eurokrise wurden sodann den unterschiedlichen Solidaritätsmodellen zugeordnet. Sowohl der Umfang und die Häufigkeit der vorgeschlagenen und beschlossenen Solidarmaßnahmen als auch ihre Rechtfertigungsargumente deuten zunehmend auf ein distributives Solidaritätsverständnis hin, wodurch nun immer stärker die Solidarbedingungen hinterfragt werden. Insbesondere die heftig umstrittene Einführung von Eurobonds als Ausdruck einer distributiven europäischen Solidarität im vollen Sinne birgt trotz seines Krisenlösungspotenzials jedoch auch einige Risiken.

Auf der Ebene der nationalen Regierungen verlangt eine solche Vergemeinschaftung von Staatsanleihen institutionelle Vorrichtungen dagegen, dass in den weniger wettbewerbsfähigen Eurostaaten eine unsolide Haushaltspolitik oder demoskopiegeleitete Wahlkampfgeschenke subventioniert werden. Effektive Kontroll- und Interventionsinstanzen mit Sanktionskompetenzen zur Vermeidung von Trittbrettfahren würden allerdings die Budgethoheit der nationalen Parlamente empfindlich einschränken. Damit steht die konstitutionelle Architektur der Europäischen Union abermals zur Disposition. Die temporäre Einschränkung demokratischer Kontrolle in einer akuten Krise kann zwar in Hobbesscher Manier mit Verweis auf die Output-Legitimation vorübergehend gerechtfertigt werden, doch ein dauerhafter, die europäische Gewaltenordnung restrukturierender Interventionsmechanismus muss demokratisch legitimiert werden (vgl. Habermas 2011). Angesichts der erheblichen distributiven Effekte dieser konstitutionellen Neuordnung stellt sich die bekannte Frage nach dem Demokratiedefizit der EU drängender denn je.

Auf der Ebene der Unionsbürger erfordert das Prinzip der Eigenverantwortlichkeit zudem eine Debatte über eine stärkere sozial- und fiskalpolitische Harmonisierung zur Angleichung der Arbeits- und Lebensbedingungen. So verweigerte beispielsweise die Slowakei bereits 2010 eine Beteiligung an den Rettungspaketen für Griechenland mit dem Hinweis auf den geringeren Wohlstand der eigenen Bürger; in Deutschland wurde hingegen diskutiert, inwiefern es einer Angleichung des gesetzlichen Renteneintrittsalters zwischen den Eurostaaten bedarf. ${ }^{32}$ Letztlich müssen die Bürger in allen Eurostaaten - sowohl der Nehmer- als auch der Geberstaaten - die Bedingungen einer distributiven Solidarität als fair akzeptieren können. Anderenfalls zerbricht eine intergouvernementale distributive Solidarität an ihrer Grundvoraussetzung, dass die Regierungen ihre Beschlüsse auch innenpolitisch rechtfertigen und durchsetzen können.

32 Allerdings liegt das reale Renteneintrittsalter in den bedrohten Eurostaaten tatsächlich über dem Durchschnitt in der EU, vgl. z. B. ZEIT ONLINE (2011b). 


\section{Literatur}

Aidukaite, Jolanta. 2009. Old Welfare State Theories and New Welfare Regimes in Eastern Europe. Challenges and Implications. Communist and Post-Communist Studies 42:2339.

Anderson, Benedict. 1983. Imagined Communities. Reflections on the Origin and Spread of Nationalism. London u. a.: Verso.

Arts, Wil, und John Gelissen. 2002. Three Worlds of Welfare Capitalism or More? A Stateof-the-Art Report. Journal of European Social Policy 12:137-158.

Athanassiou, Phoebus. 2009. Withdrawal and Expulsion from the EU and EMU. Some Reflections. European Central Bank Working Paper 10. Frankfurt a. M.: Europäische Zentralbank.

Barroso, José Manuel. 2012. Europa am Scheideweg. Perspektiven und Chancen eines geprüften Kontinents. Die Politische Meinung 1:5-12.

Baum, Rainer C. 1975. The System of Solidarities. A Working Paper in General Action Analysis. Indian Journal of Sociology 16:306-353.

Baumann, Florian. 2008. Energiesolidarität als Instrument der Versorgungssicherheit. CAP Aktuell 6. München: Centrum für angewandte Politikforschung.

Baurmann, Michael. 1998. Solidarität als soziale Norm und als Norm der Verfassung. In Solidarität. Begriff und Problem, Hrsg. Kurt Bayertz, 345-388. Frankfurt a. M.: Suhrkamp.

Bayertz, Kurt. 1998a. Solidarity and the Welfare State: Some Introductory Considerations. Ethical Theory and Moral Practice 1:293-296.

Bayertz, Kurt. 1998b. Begriff und Problem der Solidarität. In Solidarität. Begriff und Problem, Hrsg. Kurt Bayertz, 345-388. Frankfurt a. M.: Suhrkamp.

Bazant, Ursula, und Klaus Schubert. 2008. Europäische Wohlfahrtssysteme. Vielfalt jenseits bestehender Kategorien. In Europäische Wohlfahrtssysteme. Ein Handbuch, Hrsg. Ursula Bazant, Simon Hegelich und Klaus Schubert, 623-645. Wiesbaden: VS Verlag für Sozialwissenschaften.

Begg, Iain. 2005. Rethinking the Social Dimension of the EU: The Costs of Non-Social Policy. In Soziales Europa? Perspektiven des Wohlfahrtsstaates im Kontext von Europäisierung und Globalisierung, Hrsg. Alexandra Baum-Ceisig und Anne Faber, 293312. Wiesbaden: VS Verlag für Sozialwissenschaften.

Beichelt, Timm, und Nicolai von Ondarza. 2011. Von der Schulden- zur Integrationskrise? Euro-Krisenmanagement und europäische Integrationsdynamik. In Die EU auf dem Weg zur Wirtschaftsregierung? Europäische Reaktionen auf die Finanz-, Wirtschaftsund Schuldenkrise, MES-Perspektiven 1/2011, Hrsg. Timm Beichelt, Nicolai von Ondarza und Günter Verheugen, 15-21. Frankfurt/Oder: Europa-Universität Viadrina.

Beitz, Charles R. 1979. Political Theory and International Relations. Princeton: Princeton University Press.

Berger, Cathleen, und Christina Ücker. 2011. Die Finanz-, Wirtschafts- und Schuldenkrise und die Europäische Wirtschaftsregierung. In Die EU auf dem Weg zur Wirtschaftsregierung? Europäische Reaktionen auf die Finanz-, Wirtschafts- und Schuldenkrise, MES-Perspektiven 1/2011, Hrsg. Timm Beichelt, Nicolai von Ondarza und Günter Verheugen, 7-14. Frankfurt/Oder: Europa-Universität Viadrina. 
Bieler, Andreas. 2007. Der Kampf für eine soziales Europa: Gewerkschaften und die neoliberale Umstrukturierung der Europäischen Union. Kurswechsel 1:6-15.

Bundesfinanzministerium. 2011. Bundesfinanzminister Dr. Wolfgang Schäuble im Interview mit dem Deutschlandradio. http//:www.bundesfinanzministerium.de/nn_88146/ DE/Presse/Reden-und-Interviews/20110712-Deutschlandfunk.html. Zugegriffen: 29.10.2011.

Bundesregierung. 2007. Starker Binnenmarkt. Chance für Europa und Deutschland. http:// www.bundesregierung.de/Content/DE/Magazine/emags/economy/045/sp-3-starkerbinnenmarkt-chance-fuer-europa-und-deutschland.html. Zugegriffen: 29.10.2011.

Buschak, Willy. 2003. Der Europäische Gewerkschaftsbund und die Europäischen Gewerkschaftsverbände. In Europäische Gewerkschaftsorganisationen. Bestände im Archiv der sozialen Demokratie und in der Bibliothek der Friedrich-Ebert-Stiftung, Hrsg. Friedrich-Ebert-Stiftung, 2. erw. Aufl., 9-19. Bonn: Friedrich-Ebert-Stiftung.

CDU Landesvorstand Nordrhein-Westphalen. 2011. Mehr Europa im deutschen Interesse. Beschluss vom 19.09. http://www.cdu-nrw.de/images/stories/docs/Mehr_Europa_im_ deutschen_Interesse-Beschluss-LaVo20110919.pdf. Zugegriffen: 29.10.2011.

cdu.de. 2011. Regierungserklärung: Europa gelingt nur gemeinsam. http://www.cdu.de/ portal2009/26423_32593.htm. Zugegriffen: 26.04.2012.

Chwaszcza, Christine. 2008. Sozialstaatlichkeit und demokratische Legitimation in Europa. In Gerechtigkeit in Europa. Transnationale Dimension einer normativen Grundfrage, Hrsg. Helmut König, Emanuel Richter und Sabine Schielke, 119-134. Bielefeld: transcript.

Contogeorgis, Giorgios. 1978. The Greek View of the Community and Greece's Approach to Membership. In A Community of Twelve? The Impact of Further Enlargement on the European Community, Hrsg. William Wallace und Inneke Herreman, 22-31. Bruges: De Tempel.

Council of the European Union. 2011. Statement by the Heads of State or Government of the Euro Area and EU Institutions. http://www.consilium.europa.eu/uedocs/cms_data/ docs/pressdata/en/ec/123978.pdf. Zugegriffen: 29.10.2011.

Delpla, Jacques, und Jakob von Weizsäcker. 2011. Eurobonds: The Blue Bond Concept and its Implications. Bruegel Policy Contribution 2/2011. Brüssel: Bruegel.

Deppe, Frank. 2005. Der Umbau des Sozialstaates in Europa und die Probleme der Gewerkschaften. In Soziales Europa? Perspektiven des Wohlfahrtsstaates im Kontext von Europäisierung und Globalisierung, Hrsg. Alexandra Baum-Ceisig und Anne Faber, 78-94. Wiesbaden: VS Verlag für Sozialwissenschaften.

Derpmann, Simon. 2009. Solidarity and Cosmopolitanism. Ethical Theory and Moral Practice 12:303-315.

Deutsch, Franziska. 2005. Legitimacy and Identity in the European Union: Empirical Findings from the Old Member States. In European Identity. Theoretical Perspectives and Empirical Insights, Hrsg. Ireneusz Pawel Karolewski und Viktoria Kaina, 149-178. Münster: LIT.

Deutsch, Karl. 1953. Nationalism and Social Communication: An Inquiry into the Foundations of Nationality. New York u. a.: The Technology Press of the Massachusetts Institute of Technology.

Deutscher Bundestag. 2010. Plenarprotokoll 17/30.

Deutscher Bundestag. 2011. Plenarprotokoll 17/135. 
Durkheim, Émile. [1893] 1977. Über soziale Arbeitsteilung. Studie über die Organisation höherer Gesellschaften. Frankfurt a. M.: Suhrkamp.

Dworkin, Richard. 1981. What is Equality? Part II: Equality of Resources. Philosophy and Public Affairs 10:283-345.

Eder, Klaus, Kai-Uwe Hellmann, und Hans-Jörg Trenz. 1998. Regieren in Europa jenseits öffentlicher Legitimation? Eine Untersuchung zur Rolle von politischer Öffentlichkeit in Europa. In Regieren in entgrenzten Räumen. Politische Vierteljahresschrift, Sonderheft 29, Hrsg. Beate Kohler-Koch, 321-344. Opladen: Westdeutscher Verlag.

Esping-Andersen, Gøsta. 1990. The Three Worlds of Welfare Capitalism. Cambridge: Polity Press.

EUobserver. 2010. Merkel Backs Creation of European Credit Rating Agency. http://euobserver.com/19/30001. Zugegriffen: 29.10.2011.

Europäische Kommission. 2011. Grünbuch der Europäischen Kommission über die Durchführbarkeit der Einführung von Stabilitätsanleihen, Memo/11/820, 23.11.2011. http:// europa.eu/rapid/press-release_MEMO-11-820_de.htm. Zugegriffen: 14.12.2012.

European Commision, European Central Bank und International Monetary Fund. 2011. Statement by the European Commission, the ECB and IMF on the Fifth Review Mission in Greece. http:/www.imf.org/external/np/sec/pr/2011/pr11359.htm. Zugegriffen: 29.10.2011.

Eurostat. 2011. Energy, Transport and Environment Indicators. Luxembourg: Publications Office of the European Union.

Fenger, H. J. M. 2007. Welfare Regimes in Central and Eastern Europe: Incorporating Post-Communist Countries in a Welfare Regime Typology. Contemporary Issues and Ideas in Social Sciences 3:1-30.

Ferrera, Maurizio. 1996. The "Southern" Model of Welfare in Social Europe. Journal of European Social Policy 6:17-37.

Fiegle, Thomas. 2002. Von der Solidarité zur Solidarität. Ein französisch-deutscher Begriffstransfer. Münster u. a.: LIT.

FT.com. 2010. Lagarde Criticises Berlin Policy. http://cachef.ft.com/cms/s/0/ 225bbcc4-2f82-11df-9153-00144feabdc0.html\#axzz1cfe4hRHA. Zugegriffen: 29.10.2011.

Fournier, Marcel. 2005. Durkheim's Life and Context: Something New About Durkheim? In The Cambridge Companion to Durkheim, Hrsg. Jeffrey C. Alexander und Philip Smith, 41-69. Cambridge: University Press.

Ganßmann, Heiner. 2010. Soziale Sicherheit durch die EU? Staatstheoretische und europasoziologische Perspektiven. In Gesellschaftstheorie und Europapolitik: sozialwissenschaftliche Ansätze zur Europaforschung, Hrsg. Monika Eigmüller und Steffen Mau, 329-352. Wiesbaden: VS Verlag für Sozialwissenschaften.

Garrigues, Emilio. 1977. Stellungnahme zum Gesuch Spaniens um Beitritt zu den Europäischen Gemeinschaften. Europa-Archiv 19:533-536.

Geden, Oliver. 2007. Energiesolidarität im EU-Reformvertrag. Ein zentraler Baustein der europäischen Energiepolitik. SWP-Aktuell 34. Berlin: Stiftung Wissenschaft und Politik.

Gerhards, Jürgen. 1993. Westeuropäische Integration und die Schwierigkeiten der Entstehung einer europäischen Öffentlichkeit. Zeitschrift für Soziologie 22:96-110. 
Göbel, Andreas, und Eckart Pankoke. 1998. Grenzen der Solidarität. Solidaritätsformeln und Solidaritätsformen im Wandel. In Solidarität. Begriff und Problem, Hrsg. Kurt Bayertz, 463-494. Frankfurt a. M.: Suhrkamp.

Götz, Roland. 2009. Pipeline-Popanz. Irrtümer der europäischen Energiedebatte. OSTEUROPA 59:5-20.

Habermas, Jürgen. 2011. Wie demokratisch ist die EU? Die Krise der Europäischen Union im Licht einer Konstitutionalisierung des Völkerrechts. Blätter für deutsche und internationale Politik 8:37-48.

Halldenius, Lena. 1998. Non-Domination and Egalitarian Welfare Politics. Ethical Theory and Moral Practice 1:335-353.

Hartwig, Ines. 2005. Herausforderungen an die europäische Solidarität. Die Reform der EU-Strukturpolitik. In Solidarität und Beitragsgerechtigkeit. Die Reform der EU-Strukturfonds und die Finanzielle Vorausschau, Hrsg. Ines Hartwig und Wolfgang Petzold, 159-168. Baden-Baden: Nomos.

Heidenreich, Martin. 2007. The Development of Social Inequalities in Europe. In European Solidarities. Tensions and Contentions of a Concept, Hrsg. Lars Magnusson und Bo Stråth, 33-54. Brüssel: Peter Lang.

Hendricks, Jon, und C. Breckinridge Peters. 1973. The Ideal Type and Sociological Theory. Acta Sociologica 16:31-40.

Höffe, Otfried. 2002. Demokratie im Zeitalter der Globalisierung. München: C.H. Beck.

Honneth, Axel. 1991. Kampf um Anerkennung. Zur moralischen Grammatik sozialer Konflikte. Frankfurt a. M.: Suhrkamp.

Hühn, Melanie, Dörte Lerp, Knut Petzold und Miriam Stock. 2010. In neuen Dimensionen denken? Einführende Überlegungen zu Transkulturalität, Transnationalität, Transstaatlichkeit und Translokalität. In Transkulturalität, Transnationalität, Transstaatlichkeit, Translokalität. Theoretische und empirische Begriffsbestimmungen, Hrsg. Melanie Hühn, Dörte Lerp, Knut Petzold und Miriam Stock, 11-46. Münster u. a.: LIT.

Imbusch, Peter, und Dieter Rucht. 2005. Integration und Desintegration in modernen Gesellschaften. In Integrationspotenziale einer modernen Gesellschaft, Hrsg. Wilhelm Heitmeyer und Peter Imbusch, 13-71. Wiesbaden: VS Verlag für Sozialwissenschaften.

Jagodziński, Romuald. 2007. Involving European Works Councils in Transnational Negotiations - A Positive Functional Advance in their Operation or Trespassing? Industrielle Beziehungen 14:316-333.

Kant, Immanuel. [1795] 1984. Zum Ewigen Frieden. Ein philosophischer Entwurf. Stuttgart: Reclam.

Kantner, Cathleen. 2004. Kein modernes Babel. Kommunikative Voraussetzungen europäischer Öffentlichkeit. Wiesbaden: VS Verlag für Sozialwissenschaften.

Karolewski, Ireneusz P. 2005. Citizenship and Collective Identity in Europe? In European Identity: Theoretical Perspectives and Empirical Insights, Hrsg. Ireneusz P. Karolewski und Viktoria Kaina, 209-241. London: LIT.

Kersting, Wolfgang. 1998. Internationale Solidarität. In Solidarität. Begriff und Problem, Hrsg. Kurt Bayertz, 411-429. Frankfurt a. M.: Suhrkamp.

Kluge, Susann. 1999. Empirisch begründete Typenbildung. Zur Konstruktion von Typen und Typologien in der qualitativen Sozialforschung. Opladen: Leske + Budrich.

Koselleck, Reinhart. 1979. Bund. Bündnis, Föderalismus, Bundesstaat. In Geschichtliche Grundbegriffe. Historisches Lexikon zur politisch-sozialen Sprache in Deutschland, 
Hrsg. Reinhart Koselleck, Otto Brunner, und Werner Conze, Bd. 1, 582-671. Stuttgart: Klett-Cotta.

Kymlicka, Will. 1990. Contemporary Political Philosophy. An Introduction. Oxford: Clarendon Press.

Lessenich, Stephan. 2010. Der „Wohlfahrtsstaat Europa“ zwischen Wunsch und Wirklichkeit. In Gesellschaftstheorie und Europapolitik: sozialwissenschaftliche Ansätze zur Europaforschung, Hrsg. Monika Eigmüller und Steffen Mau, 321-328. Wiesbaden: VS Verlag für Sozialwissenschaften.

Leibfried, Stephan. 1992. Towards a European Welfare State? On Integrating Poverty Regimes into the European Community. In Social Policy in a Changing Europe, Hrsg. Zsuzsa Ferge und Jon E. Kolberg, 245-279. Frankfurt a. M.: Campus.

Lindbekk, Tore. 1992. The Weberian Ideal-type: Development and Continuities. Acta Sociologica 35:285-297.

Luhmann, Niklas. 1992. Arbeitsteilung und Moral. Durkheims Theorie. In: Emile Durkheim, Über soziale Arbeitsteilung. Studie über die Organisation höherer Gesellschaften, 19-40. Frankfurt a. M.: Suhrkamp.

Magnusson, Lars, und Bo Stråth. 2007. A Social Polity? Challenges to European Inequalities, In European Solidarities - Tensions and Contentions of a Concept, Hrsg. Lars Magnusson und Bo Stråth, 11-31. Brüssel: Peter Lang.

Marek, Norbert. 2010. Diskurs der Geopolitik - Diskurs der Sprachlosigkeit. PolnischRussische Schwierigkeiten. European Journal of Transnational Studies 2:30-45.

Mau, Steffen. 2009. Europäische Solidarität. Erkundung eines schwierigen Geländes. In Solidarität und internationale Gemeinschaftsbildung. Beiträge zur Soziologie der internationalen Beziehungen, Hrsg. Sebastian Harnisch, Hanns W. Maull und Siegfried Schieder, 63-87. Frankfurt a. M./New York: Campus.

Metz, Karl H. 1998. Solidarität und Geschichte. Institutionen und sozialer Begriff der Solidarität in Westeuropa im 19. Jahrhundert. In Solidarität. Begriff und Problem, Hrsg. Kurt Bayertz, 172-194. Frankfurt a. M.: Suhrkamp.

Müller, Hans-Peter, und Michael Schmid. 1992. Arbeitsteilung, Solidarität und Moral. Eine werksgeschichtliche und systematische Einführung in die „Arbeitsteilung” von Emile Durkheim. In: Emile Durkheim, Über soziale Arbeitsteilung. Studie über die Organisation höherer Gesellschaften, 481-521. Frankfurt a. M.: Suhrkamp.

Münkler, Herfried. 2004. Enzyklopädie der Ideen der Zukunft: Solidarität. In Transnationale Solidarität. Chancen und Grenzen, Hrsg. Jens Beckert, Julia Eckert, Martin Kohli und Wolfgang Streeck, 15-28. Frankfurt a. M.: Campus.

Papineau, David. 1976. Ideal Types and Empirical Theories. British Journal for the Philosophy of Science 27:137-146.

Pogge, Thomas. 1994. An Egalitarian Law of Peoples. Philosophy and Public Affairs 23:195-224.

Pöttering, Hans-Gert. 2012. Den Gemeinschaftsgeist erneuern. Europa benötigt Fairness und Solidarität. Die Politische Meinung 1:13-18.

Preuß, Ulrich K. 1998. Nationale, supranationale und internationale Solidarität. In Solidarität. Begriff und Problem, Hrsg. Kurt Bayertz, 399-410. Frankfurt a. M.: Suhrkamp. Rawls, John. [1971] 1975. Eine Theorie der Gerechtigkeit. Frankfurt a. M.: Suhrkamp. Rawls, John. 1999. The Law of Peoples. Cambridge u. a.: Harvard University Press. 
Reding, Viviane. 2011. The end of Europe? No, the beginning of a stronger, more united Europe. http://europa.eu/rapid/pressReleasesAction.do? reference=SPEECH/11/566\&fo rmat=HTML\&aged=0\&language=EN\&guiLanguage=en. Zugegriffen: 29.10.2011.

Rehn, Olli. 2010. A Toolbox for Stronger Economic Governance in Europe. http://europa. eu/rapid/pressReleasesAction.do? reference=MEMO/10/288\&format=HTML\&aged $=0$ \&language $=E N \&$ guiLanguage $=$ en. Zugegriffen: 29.10.2011.

Risse, Thomas. 2002. Zur Debatte um die (Nicht-)Existenz einer europäischen Öffentlichkeit - Was wir wissen, und wie es zu interpretieren ist. Berliner Debatte Initial 13:15-23.

Saryusz-Wolski, Jacek. 1994. The Reintegration of the 'Old Continent': Avoiding the Costs of 'Half Europe'. In Economic and Political Integration in Europe. Internal Dynamics and Global Context, Hrsg. Simon Bulmer und Andrew Scott, 19-28. Oxford: University Press.

Scharpf, Fritz. 2002. The European Social Model. Coping with the Challenges of Diversity. Journal of Common Market Studies 40:645-670.

Scharpf, Fritz. 2009. Weshalb die EU nicht zur sozialen Marktwirtschaft werden kann. Zeitschrift für Staats- und Europawissenschaften 7:419-435.

Scharpff, Nancy. 2012. Von der „sozialen Dimension Europas“ zum „sozialen Europa“. Sozialpolitikentwicklung in der Globalisierung. In Zwischen Gemeinschaft und Gesellschaft. Sozialpolitik in historisch-soziologischer Perspektive, Hrsg. Monika Eigmüller, 177-201. Weinheim/Basel: Beltz Juventa.

Schieder, Siegfried. 2009. Zur Theorie der Solidarität und internationalen Gemeinschaft. In Solidarität und internationale Gemeinschaftsbildung. Beiträge zur Soziologie der internationalen Beziehungen, Hrsg. Sebastian Harnisch, Hanns W. Maull und Siegfried Schieder, 11-59. Frankfurt a. M./New York: Campus.

Schmid, Michael. 1989. Solidarität und Arbeitsteilung. Bemerkungen zu Durkheims Theorie. In Kultur und Gesellschaft, Hrsg. Max Haller, 518-531. Frankfurt a. M. u. a.: Campus.

Schuyt, Kees. 1998. The Sharing of Risks and the Risks of Sharing: Solidarity and Social Justice in the Welfare State. Ethical Theory and Moral Practice 1:297-311.

SPIEGEL ONLINE. 2010. Kampf gegen Währungskrise. Trichet fordert "Quantensprung” von Euro-Staaten. http://www.spiegel.de/politik/ausland/0,1518,694891,00.html. Zugegriffen: 29.10.2011.

Stawarska, Renata. 1999. EU Enlargement from the Polish Perspective. Journal of European Public Policy 6:822-838.

Stephan, Antje. 2005. Europäische Beschäftigungsstrategie und die offene Methode der Koordinierung. Entstehung und Bedeutung für Politiksteuerung auf EU-Ebene. In Soziales Europa? Perspektiven des Wohlfahrtsstaates im Kontext von Europäisierung und Globalisierung, Hrsg. Alexandra Baum-Ceisig und Anne Faber, 264-292. Wiesbaden: VS Verlag für Sozialwissenschaften.

Stinchcombe, Arthur. 1968. Constructing Social Theories. New York: Harcourt Brace.

Streeck, Wolfgang. 2000. Competitive Solidarity: Rethinking the ,European Social Model'. In Kontingenz und Krise. Institutionenpolitik in kapitalistischen und postsozialistischen Gesellschaften, Hrsg. Karl Hinrichs, Herbert Kitschelt und Helmut Wiesenthal, 245-261. Frankfurt a. M./New York: Campus. 
Tagesspiegel Online. 2011. Angela Merkel im Interview. „Die Sehnsucht nach einfachen Lösungen ist groß“. http://www.tagesspiegel.de/politik/die-sehnsucht-nach-einfachenloesungen-ist-gross/4596984.html. Zugegriffen: 29.10.2011.

Taylor, Charles. 1993. Multikulturalismus und die Politik der Anerkennung. Frankfurt a. M.: Fischer.

Thome, Helmut. 1998. Soziologie und Solidarität: Theoretische Perspektiven für die empirische Forschung. In Solidarität. Begriff und Problem, Hrsg. Kurt Bayertz, 217-262. Frankfurt a. M.: Suhrkamp.

Traum, Detlef. 2005. Europäische Betriebsräte. Eine empirische und theoretische Analyse aus der Perspektive der Systemtheorie. München/Mering: Rainer Hampp.

Vetters, Regina, Erik Jentges und Hans-Jörg Trenz. 2009. Whose Project is it? Media Debates on the Ratification of the EU Constitutional Treaty. Journal of European Public Policy 16:412-430.

Voland, Eckart. 1998. Die Natur der Solidarität. In Solidarität. Begriff und Problem, Hrsg. Kurt Bayertz, 297-318. Frankfurt a. M.: Suhrkamp.

Wagener, Hans-Jürgen. 2009. Zwischen Solidarität und Subsidiarität. Was ist die soziale Kompetenz der Europäischen Union. In Bedingungen europäischer Solidarität, Hrsg. Matthias T. Vogt, Jan Sokol, Beata Ociepka, Detlef Pollack und Beata Mikołajczyk, 6589. Frankfurt a. M.: Peter Lang.

Waldron, Jeremy. 1987. Theoretical Foundations of Liberalism. Philosophical Quarterly 37:463-482.

Weber, Max. 1951 [1904]. Die „Objektivität“ sozialwissenschaftlicher und sozialpolitischer Erkenntnis. In: ders., Gesammelte Aufsätze zur Wissenschaftslehre, 2. Aufl., 146214. Tübingen: J. C. B. Mohr.

Wendt, Alexander. 1999. Social Theory of International Politics. Cambridge u. a.: Cambridge University Press.

Wildt, Andreas. 1998. Solidarität - Begriffsgeschichte und Definition heute. In Solidarität. Begriff und Problem, Hrsg. Kurt Bayertz, 202-216. Frankfurt a. M.: Suhrkamp.

WirtschaftsBlatt. 2011. Brüssel erteilt europäischer Ratingagentur Absage. http://www. wirtschaftsblatt.de/home/international/wirtschaftspolitik/bruessel-erteilt-europaeischerratingagentur-absage-486951/index.do. Zugegriffen: 29.10.2011.

WirtschaftsWoche Online. 2011. Wolfgang Schäuble: „Griechenland braucht Zeit - und unsere Hilfe“. http:/www.wiwo.de/politik-weltwirtschaft/griechenland-braucht-zeitund-unsere-hilfe-482574. Zugegriffen: 29.10.2011.

Wonka, Arndt. 2007. Um was geht es? Konzeptspezifikation in der politikwissenschaftlichen Forschung. In Forschungsdesign in der Politikwissenschaft. Probleme-Strategien - Anwendungen, Hrsg. Thomas Gschwend und Frank Schimmelfennig, 63-89. Frankfurt a. M./New York: Campus.

ZEIT ONLINE. 2010. IWF-Chef fürchtet Domino-Effekt in der EU. http://www.zeit.de/ wirtschaft/2010-05/griechenland-krise-iwf. Zugegriffen: 29.10.2011.

ZEIT ONLINE. 2011a. Von der Leyen will Vereinigte Staaten von Europa. http://www. zeit.de/politik/deutschland/2011-08/leyen-politische-union-europa. Zugegriffen: 29.10.2011.

ZEIT ONLINE. 2011b. Faul und wohlhabend. http://www.zeit.de/2011/25/ArbeitsweltDeutschland. Zugegriffen: 29.10.2011. 
Zürcher, Markus D. 2001. Transnationale Solidarität. Grundlage für ein soziales Europa. In Solidarität und Selbstverwirklichung, Hrsg. Joachim Küchehoff, 57-72. Gießen: Psychosozial-Verlag.

\section{Autorenangaben}

Prof. Dr. Heinz Kleger,

Universität Potsdam, Wirtschafts- und Sozialwissenschaftliche Fakultät, Lehrstuhl für Politische Theorie, August-Bebel-Straße 89, 14482 Potsdam, kleger@uni-potsdam.de

[Korrespondenzautor]

Thomas Mehlhausen, M.A., Universität Potsdam, Wirtschafts- und Sozialwissenschaftliche Fakultät, Lehrstuhl für Politische Theorie, August-Bebel-Straße 89, 14482 Potsdam, mehlhaus@uni-potsdam.de [Korrespondenzautor] 